\title{
Emphysematous Cystitis - A Case Report
}

\author{
Carla Meira, Ana Jerónimo, Carlos Oliveira, Augusta Amaro and Cristina Granja \\ Intensive Care Department, Pedro Hispano Hospital; Matosinhos, Portugal
}

\begin{abstract}
Emphysematous Cystitis is a primary infection of the bladder with production of gas by bacteria. The infection is uncommon, still has obvious clinical importance due to its morbidity and mortality potential, as the following case enlightens. We report a clinical case of a patient admitted with acute myocardial infarction who developed an acute emphysematous cystitis, a further complication in his long and complex period of hospitalization.

Key-Words: Emphysematous cystitis, urinary tract infections.
\end{abstract}

Emphysematous Cystitis is a primary infection of the bladder with production of gas by bacteria. It is usually diagnosed in diabetic patients with poor glycemic control or in immunocompromised. The severity of the clinical picture varies from simple cystitis-like symptoms to full-blown sepsis. Therefore, a high index of clinical suspicion is essential in an early phase. The appropriate antibiotic therapy is usually curative, but in more complex cases surgery may be required [1]. The infection is uncommon, still has obvious clinical importance due to its morbidity and mortality potential, as the following case enlightens. We report a clinical case of a patient admitted with acute myocardial infarction who developed an acute emphysematous cystitis, a further complication in his long and complex period of hospitalization.

\section{Case Report}

A 81-year-old caucasian male with cardiovascular hypertension and dyslipemia was admitted to the emergency ward with non ST-segment elevation acute myocardial infarction. After acute phase treatment, a coronarography showed two vessels disease and he underwent an angioplasty of the right coronary with implantation of a Cypher stent. The patient had a good recovery in the initial stage, but its condition worsened during the hospitalization and he developed acute lung edema. The echocardiography showed serious mitral insufficiency resulting from rupture of the papillary muscle. A cardiac operation was performed in order to replace the mitral valve for a biologic prothesis and implantation of venous bypass graft to the anterior descending coronary artery. The operation went without incidents. The patient continued with oral anticoagulants and the remaining medication. Urinary catheterization was performed during the postoperative period.

Seven days after surgery his condition worsened again with deterioration in the general state and prostration in association with the appearance of haematuria. On physical examination he presented dehydrated, pale, sweaty, tachypneic Received on 20 June 2008; revised 13 November 2008.

Address for correspondence: Dr.Carla Cristina do Rego Meira. Rua do Barroco, $n^{\circ}$ 84, Vila Nova de Anha. Zip code: 4900 - Viana do Castelo. Portugal. E-mail: carlameira@yahoo.com. Telefone: 00351 964764720 .

The Brazilian Journal of Infectious Diseases

2008;12(6):552-554. (C) 2008 by The Brazilian Journal of Infectious Diseases and Contexto Publishing. All rights reserved. with O2 Saturation: 84\% (FiO2: 0.6), signs of poor peripheral perfusion. Vital signs: temperature: $37^{\circ}$ centigrade, blood pressure: 80/40 mm Hg, heart rate: $80 \mathrm{bpm}$. Cardiopulmonary auscultation showed tachycardia, no other abnormalities. Abdomen was soft and depressible, no pain on palpation. Biochemical analysis showed increase in inflammatory markers and acute renal failure: Haemoglobin: 14 g/dL; Leukocyte: 25 x103 $\mu / L$; Neutrophils: 91\%; Platelet: $188 \times 10^{3} \mu / L$; Urea/ Creatinine: 161/4 mg/dL; Na+ 132 mEq/L, K+ 5.9 mEq/L, CReactive Protein: $29 \mathrm{mg} / \mathrm{dL}$; Coagulation study was abnormal: aPTT: 51; INR: 5.9; Blood gas analysis was as follow: $\mathrm{pH} 7.51$; PCO2 15 mmHg; PO2 115 mmHg; HCO3 12 mmol/L; Lactates: $6.6 \mathrm{mmol} / \mathrm{L}$. A nosocomial urinary tract infection with severe sepsis was assumed as the diagnosis. Microbiological products were collected and the patient was empirically treated with piperacillin/tazobactam. Abnormal coagulation was corrected with vitamin K. Echocardiogram was repeated to exclude cardiac complications: valvular prothesis in mitral position had normal function, systolic function of the left ventriculus was preserved and no other relevant changes were detected. Initial aggressive treatment with fluids and antibiotic was not successful and the patient developed septic shock being admitted to the intensive care unit with multiorganic dysfunction. Haematuria was increasing and abdominal pain was now present. He was started on mechanical ventilation and resuscitation with volume and amines. An abdominal scan revealed gas in the excretory urinary tracts and bladder. A further explanation for the images was necessary and a CTscan was performed. Multiple gas pockets along the vesical wall were present, and the images were compatible with the diagnosis of emphysematous cystitis (Figure 1). Enterobacter aerogenes was isolated in urine culture and hemoculture. Subsequently antibiotic therapy was substituted for Ciprofloxacin, in accordance with antibiogram.

The clinical evolution was unfavourable during the first days in the intensive care unit, but it slowly and gradually improved. The patient was ready for ventilator weaning and extubation on the second week. Hemodynamic support with amines was stopped. The urine culture was taken once again and presented sterile. When clinically stable, he was transferred to the intermediate care unit, where his condition continued slowly improving. After 10 days a new worsening of his clinical condition was present, with persistent fever and respiratory complaints, compatible with a nosocomial 
Figure 1. CT scan of the pelvis revealing gas in the bladder and the bladder wall.

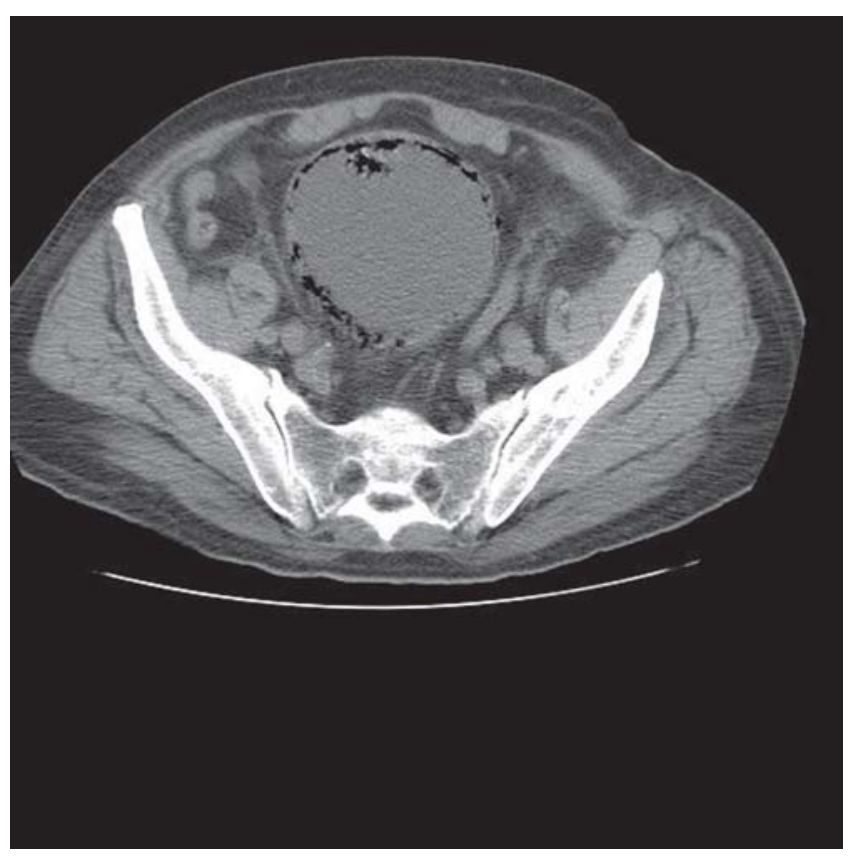

respiratory infection. New courses of empirical large spectrum antibiotics were started, with no clinical improvement. Microbiologic results were negative. After discussion of the poor prognosis with the patient's family, it was clear that there was no benefit in proceeding with invasive resuscitation manoeuvres and the patient died a few days after.

\section{Discussion}

Despite belonging to a low percentage of all the urinary tract infections, gas-producing infections are relevant as they may lead to death. Three categories of this type of infections are recognized: emphymatous pyelonephritis, emphymatous pyelitis or emphymatous cystitis.

Emphymatous pyelonephritis is a necrotic infection. The gas is produced in the renal and perirenal parenchyma and $90 \%$ of the cases are reported in diabetic patients. The delay on the appropriate therapy contributes to a high mortality rate, in some case series up to $80 \%$ [2].

Emphymatous cystitis is a rare disease that is mainly diagnosed in diabetic and immunocompromised patients. It is also reported to be in association with neurogenic bladder, obstruction of the urinary tracts, catheter use and chronic infections of the urinary tract.

The microorganisms most often involved in this infection are Escherichia coli and Klebsiella pneumonia and the less common are Enterobacter, Proteus, Streptococci and Candida [3]. Despite being the bacteria the most common agent, the funguses may also be responsible for this clinical picture [4].

The exact mechanism by which the gas is produced in the emphymatous infection is not quite clear. In diabetic patients, one of the reasons seams to be the production of
CO2 by the microorganisms through the fermentation of glucose, which occurs when the glucose concentration is high. Since the emphymatous infections may occur in nondiabetic patients, it has been suggested that the urinary lactulose and tissue proteins may be useful as substrate to the gas production. Another factor that may help in this process is the impaired transportation of gas due to the local inflammation or some kind of obstructive process increasing the local pression and decreasing the circulation. This may involve tissue necrosis which becomes a good culture for pathogens to produce gas [5].

The most common clinical features are fever and abdominal pain along with dysuria, haematuria and pneumaturia.

The diagnosis is provided by radiographic image. The most obvious radiographic clues are small pockets of gas in the mucous membrane of the bladder.

Other causes for the presence of air in the bladder such as fistula with the intestine or vagina, after trauma or instrumentation, have to be excluded [2].

The appropriate treatment involves endovenous antibiotic theraphy with broad-spectrum such as fluoroquinolones, penicillin with inhibitor of the beta-lactamases (imipenem, ticarcilin/clavulanat) or third-generation cefalosporines. Antifungal agents may be used (systemic or intravesical), if a fungus infection is reported [5]. The full recovery from any infection with gas production depends on early diagnosis plus correction of the subjacent causes, glycemic control, long-term therapeutic with antibiotic therapy (3 to 6 weeks) and surgery, if required [2].

The reported case shows the seriousness and the atypical presentation that this infection may assume. The diagnosis was made indeed on a non-diabetic patient in an unusual clinical situation. The diabetes mellitus and the bad glycemic control are the main risk factors for this type of infection. We considered that the indwelling urinary catheter during the hospitalization may have been the responsible factor for the infection development. The agent isolated from this patient, the Enterobacter aerogeneos, also is not reported as being the most common. The empiric broad-spectrum antibiotic (piperacilin/tazobactam) used for the nosocomial urinary tract infection was found to be ineffective. The organism was resistant according to the biogram and a therapeutic adjustment was needed. This relatively rare pathology has a high mortality and morbidity rate but despite the patient age and previous complex clinical situation, the initial conservative treatment and the support offered in the intensive care unit enabled a favourable evolution. Subsequently, the patient developed complications involving a nosocomial respiratory infection without favourable outcome which was fatal.

Interesting to reflect that this patient survived an acute myocardial infarction followed by a complex cardiac surgery but nosocomial infections were the main factors affecting his prognosis and survival. Our medical resources, especially antibiotic therapies, are still not sufficiently effective against this type of conditions. Control of risk factors and early clinical 
suspicion are important additional therapeutic measures to prevent unfavourable complications.

\section{Acknowledgements}

Sónia Miranda for translating this article.

\section{References}

1. Rasoul Mokabberi, Keyvan Ravakhah. Emphysematous Urinary Tract Infections:Diagnossis, Treatment and Survival (Case review Series). The American Journal of medical sciences 2007;333:111-5.
2. Ajay Kumarhttp, John H. Turney, Aleck M. Brownjohn, Michael $\mathrm{J}$ McMahon. Unusual bacterial infections of the urinary tract in diabetic patients-rare but frequently lethal. Nephrol Dial Transplant 2001;16:1062-5.

3. Esther Nemati, Ramen Basra, Joyce Fernandes, Jeremy B. Levy. Emphysematous cystitis. Nephrology Dialysis Transplantation 2005;20(3):652-3.

4. Marvalyn Decambre, Peter Albertsen, Scott Rutchik. Emphysematous Cystitis: Caveats of Complex Presentations. Infect in Uro 2002;15(4):19-21.

5. Grupper M., Kravtsov A., Potasman I. Emphysematous Cystitis: Illustrative Case Report and Review of the Literature. Medicine 2007;86(1):47-53. 